\title{
Automatic Brain Tumor Segmentation from MRI Images using Superpixels based Split and Merge Algorithm
}

\author{
Raghda A. Ali ${ }^{1}$, Loay K. Abood ${ }^{2}$ \\ ${ }^{1,2}$ Department of Computer, Collage of Science, University of Baghdad, Baghdad, Iraq
}

\begin{abstract}
Brain tumor segmentation, an essential but challenging task, has long attracted much attention from the medical imaging community. Recently, successful applications of sparse coding and dictionary learning has emerged in various vision problems including image segmentation. In this paper, a superpixel-based framework for automated brain tumor segmentation is introduced. A reformulation of the processes that composed the split and merge technique is proposed. A recursive split process is done first; then, after the merge process, a segmentation of the image is obtained. This is possible because the merging is implemented like a growth process, so the grouping has been eliminated. The use of a complete quad tree helps to achieve the reformulation. The determination of the region uniformity in each process is carried out with a different predicate, because of the different nature of the two processes. Experiments on a blocks world image and an industrial parts image are included to show the result of applying the algorithm.
\end{abstract}

Keywords: MRI, Brain Tumor, Segmentation, Superpixel, Split and Merge

\section{Introduction}

Brain tumor, which is one of the most common brain diseases, has affected and devastated many lives. According to International Agency for Research on Cancer (IARC) approximately, more than 126000 people are diagnosed for brain tumor per year around the world, with more than 97000 mortality rate [1]. Despite consistent efforts to overcome the problems of brain tumors, statistics still shows low survival rate of brain tumor patients. To combat this, recently, researchers are using multi-disciplinary approach involving knowledge in medicine, mathematics and computer science to better understand the disease and find more effective treatment methods.

Magnetic resonance (MR) imaging and computer tomography (CT) scanning of the brain are the two most common tests undertaken to confirm the presence of brain tumor and to identify its location for selected specialist treatment options. Currently, there are different treatment options available for brain tumor. These options include surgery, radiation therapy, and chemotherapy. The choice for the treatment options depends on the size, type, and grade of the tumor. It also dependents on whether or not the tumor is putting pressure on vital parts of the brain. Whether the tumor has spread to other parts of the central nervous system (CNS) or body, and possible side effects on the patient concerning treatment preferences and overall health [2] are important considerations when deciding the treatment options.

Accurate detection of the type of brain abnormality is highly essential for treatment planning in order to minimize diagnostic errors. The accuracy can be improved by using computer aided diagnosis (CAD) systems. The basic concept of $\mathrm{CAD}$ is to provide a computer output as a second opinion to assist radiologists' image interpretation and to reduce image reading time. This improves the accuracy and consistency of radiological diagnosis. However, segmentation of the image of brain tumors is a very difficult task. In the first place, there are a large class of tumor types which have a variety of shapes and sizes [3]. Appearance of brain tumors at different locations in the brain with different image intensities [2] is another factor that makes automated brain tumor image detection and segmentation difficult.

This paper presents superpixel based split and merge method used during brain tumor detection through MRI image segmentation.

\section{Magnetic Resonance Imaging}

Magnetic resonances imaging (MRI), nuclear magnetic resonance imaging (NMRI), or magnetic resonance tomography (MRT) are medical imaging techniques used in radiology to visualize internal structures of the body in detail. MRI makes use of the property of nuclear magnetic resonance (NMR) to image nuclei of atoms inside the body.

An MRI scanner is a device in which the patient lies within a large, powerful magnet where the magnetic field is used to align the magnetization of some atomic nuclei in the body, and radio frequency fields to systematically alter the alignment of this magnetization. MRI is of mainly 2 types:

- T1-weighted MRI Spin-lattice relaxation time.

- T2-weighted MRI Spin-spin relaxation time.

Another type of MRI is:

- T*2-weighted MRI (Contrast Enhance).

In clinical practice, MRI is used to distinguish pathologic tissue (such as a brain tumor) from normal tissue [4].

\section{What is Segmentation?}

Segmentation means division of an image into several connected regions. Basically, segmentation could be done 


\section{International Journal of Science and Research (IJSR) \\ ISSN (Online): 2319-7064}

Index Copernicus Value (2015): 78.96 | Impact Factor (2015): 6.391

with two definitions of region: A region can be defined as a group of connected similar pixels, or a set of connected pixels surrounded by discontinuities (edges). Split and merge uses the first approach.

Mathematically speaking: if the whole image is represented by the set of pixels (called R), than it should get subsets such as:

1. Segmentation is complete, so all sub-regions sum up to the whole $R$. Union of all regions is $R_{1} U R_{2} U \ldots U R_{n}=$ $\mathrm{R}$.

2. $R_{i}$ is connected.

3. Regions are distinct. $R_{i} \cap R_{j}=\varnothing$ given that $i \neq j$.

4. Regions have similar properties. This could be expressed by a function called the homogeneity criterion (P). It should give TRUE for the members of the given region, and FALSE for all the other regions.

Neighbor regions cannot be merged. For all regions $P\left(R_{i} U\right.$ $\left.\mathrm{R}_{\mathrm{j}}\right)=$ FALSE given that $\mathrm{i} \neq \mathrm{j}$.

The main task of segmentation is to automatically recognize predefined labels (i.e. human, vehicle, grass, sky and etc.) [5]. Superpixels, also known as regions in an over segmentation of the image, would be more natural and presumably lead to more efficient processing. The super pixel method had been increasingly used in image processing field, which can group pixels using the degree of feature similarity between pixels and acquire the redundant information of the image. Hence, it can greatly reduce the complexity of image post-processing tasks [6].

Superpixels are becoming increasingly popular for use in computer vision applications. Segmentation provides the partial support for computing region based features. The desired properties of superpixels segmentation depends on the application of interest. Here we list some general properties required by various vision applications [7], [8].

The following properties are generally desirable:

1) Superpixels should adhere well to image boundaries.

2) When used to reduce computational complexity as a preprocessing step, superpixels should be fast to compute, memory efficient, and simple to use.

3) When used for segmentation purposes, superpixels should both increase the speed and improve the quality of the results [9].

\section{Split and Merge Algorithm}

Fully merging methods are, mostly; computationally expensive because the initial point of such method is small regions (individual points). This method can be more efficient by recursively splitting the image into smaller and smaller regions until all individual regions are coherent, then recursively merging these to produce larger coherent regions. The steps of this algorithm are shown in figure 1.

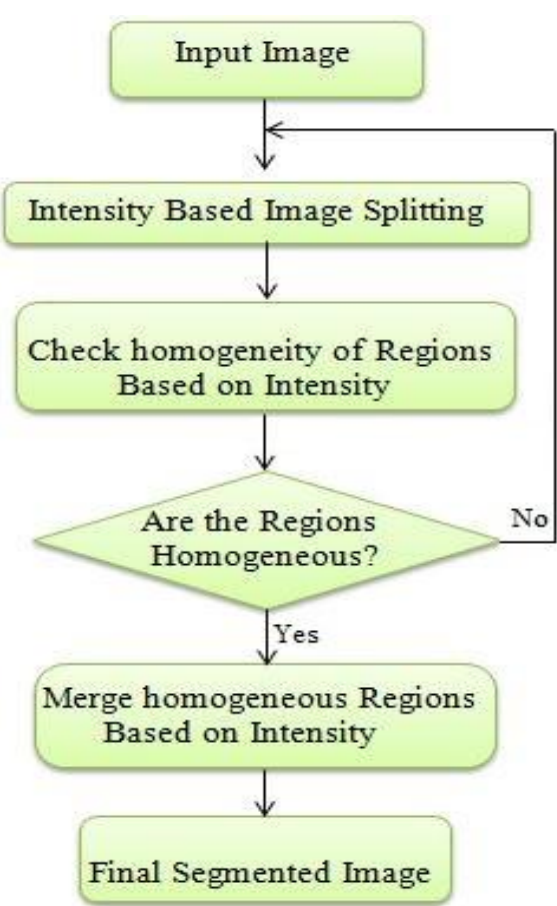

Figure 1: Split and Merge Algorithm

First, we must split the image. Start by considering the entire image as one region.

1) If the entire region is coherent (i.e., if all pixels in the region have sufficient similarity), leave it unmodified.

2) If the region is not sufficiently coherent, split it into four quadrants and recursively apply these steps to each new region.

Split-and-merge segmentation is based on a quad tree partition of an image. It is sometimes called quad tree segmentation. In this paper, a quadratic tree (split and merge) algorithm was used to locate and initialize the seed number and location through the image space rather than the old method where seeds distributed regularly as rows and columns. This method starts at the root of the tree that represents the whole image. If it is found non-uniform (not homogeneous), then it is split into four son-squares (the splitting process), and so on so forth.

Conversely, if four son-squares are homogeneous, they can be merged as several connected components (the merging process) [10]. This proposed approach isolate initially the symmetry block from other ones, which generally cluster the image more uniformly than the traditional one.

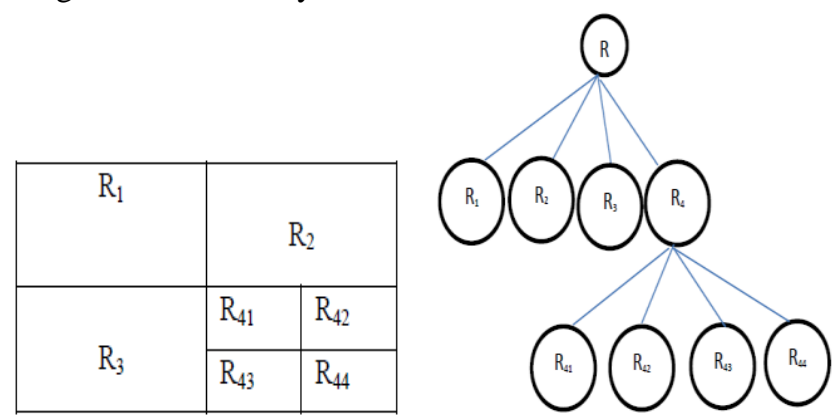

Figure 2: Partitioned image and Quad tree Image. 


\section{International Journal of Science and Research (IJSR) \\ ISSN (Online): 2319-7064}

Index Copernicus Value (2015): 78.96 | Impact Factor (2015): 6.391

\section{Pseudo Code}

Here is a pseudo-code to split and merge algorithm:

1) Initial: we have only one big region (the whole image).

2) Split: If $P\left(R_{i}\right)=T R U E$ proceed to next step. Otherwise subdivide $R_{i}$ to four sub-regions and perform step 2 on them.

3) Merge: If $R_{i}$ and $R_{j}$ are neighbors and $P\left(R_{i} U R_{j}\right)=$ TRUE, merge the two regions, than repeat step 3 . If there are no such regions we are finished.

\section{Results and Discussions}

Digital Imaging and Communications in Medicine (DICOM) is considered as benchmark database when implementing and testing many MRI segmentation systems.

In this paper the DOCOM, which had been constructed by Doctor Antoine Rosset (CEO and OsiriX company Guru), and Joris Heuberger (CTO and Problem Solver), Many cases from DICOM database are considered to evaluate the performance of the proposed method each patient Database contain 22 slice of T1-weighted MRI images and 22 slice of T2-weighted MRI [11], has been used in our experiments. Some of the 2D slice may not contain any tumor. For acquisition of single slice from the 3D image, MHA read function was used. A real dataset was also used from Oncology teaching hospital, Medical City, Baghdad, Iraq. each patient Database contain 20-30 slice of T1-weighted MRI images, 20-30 slice of T2-weighted MRI images, axial, sagittal and coronal. The proposed split and merge algorithm for image segmentation was implemented in MATLAB. Several MRI images were tested. The result shows efficient segmentation as shown in Figure 3.

\section{Conclusions}

In this paper, an automatic Superpixel based split and merge method for automatically segment 2D MRI image is proposed. The implementation results show satisfactory results for image segmentation. This implies that the proposed algorithm can be successfully used to achieve automatic image segmentation, and can be used in various MRI brain images. One of the advantages of this algorithm is not to allow user intervention. The other benefit of this algorithm is clearly that the proposed algorithm able to consuming less execution time.

\section{References}

[1] Ferlay J, Shin HR, Bray F, Forman D, Mathers C and Parkin DM, GLOBOCAN 2008 v2.0, Cancer Incidence and Mortality Worldwide, International Agency for Research on Cancer, Lyon, France, 2010.

[2] http://www.radiologyassistant.nl.

[3] Louis D.N., Ohgaki H., Wiestler O.D, Cavenee W.K. (Eds.), "WHO Classification of Tumors of the Central Nervous System", International Agency for Research on Cancer (IARC), Lyon, France, 2007.

[4] Shah, H. J., Vala, H., Rana, H. R., and Bhaidasna, Z., "Study on Various Methods for Detecting Tumor on
MRI Images", International Journal, Vol. 2, No. 2, 2014.

[5] SeungRyul Baek, Taegyu Lim, Yong Seok Heo, Sungbum Park, Hantak Kwak and Woosung Shim, "Superpixel Coherency and Uncertainty Models for Semantic Segmentation", ICCVW, P.P. 275-283, 2013.

[6] Shiyon ji,benzheng Wei, Zhen Yi,Gonping Yang and Yilong Yin, "A New Multistage Medical Segmentation Method Based on Superpixel and Fuzzy Clustering", computational and mathematical method in medicine, 2014.

[7] Radhakrishna Achanta, Appu Shaji, Kevin Smith, Aurelien Lucchi, Pascal Fua, and Sabine Süsstrunk, "SLIC Superpixels", EPFL Technical Report, no. 149300, 2010.

[8] Radhakrishna Achanta, Appu Shaji, Kevin Smith, Aurelien Lucchi, Pascal Fua, and Sabine Süsstrunk, "SLIC Superpixels Compared to State-of-the-art Superpixel Methods", IEEE Transactions on Pattern Analysis and Machine Intelligence, Vol. 34, No. 11, P.P. 2274 - 2282, 2012.

[9] Carl Yuheng Ren and Ian Reid, "A real-time implementation of SLIC superpixelSegmentation", Technical Report, 2011.

[10] S. K. Bandhyopadhya and T. U. Paul, "-Segmentation of Brain MRI Image- A Review", International Journal of Advanced Research in Computer Science and Software Engineering, Vol. 2, No. 3, pp. 2277 - 128X, 2012.

[11] Antoine and Spadola, Luca and Ratib, Osman Rosset, "OsiriX: An Open-Source Software for Navigating in Multidimensional DICOM Images," Journal of Digital Imaging, vol. 17, no. 3, pp. 205-216, 2004. 
International Journal of Science and Research (IJSR)

ISSN (Online): 2319-7064

Index Copernicus Value (2015): 78.96 | Impact Factor (2015): 6.391
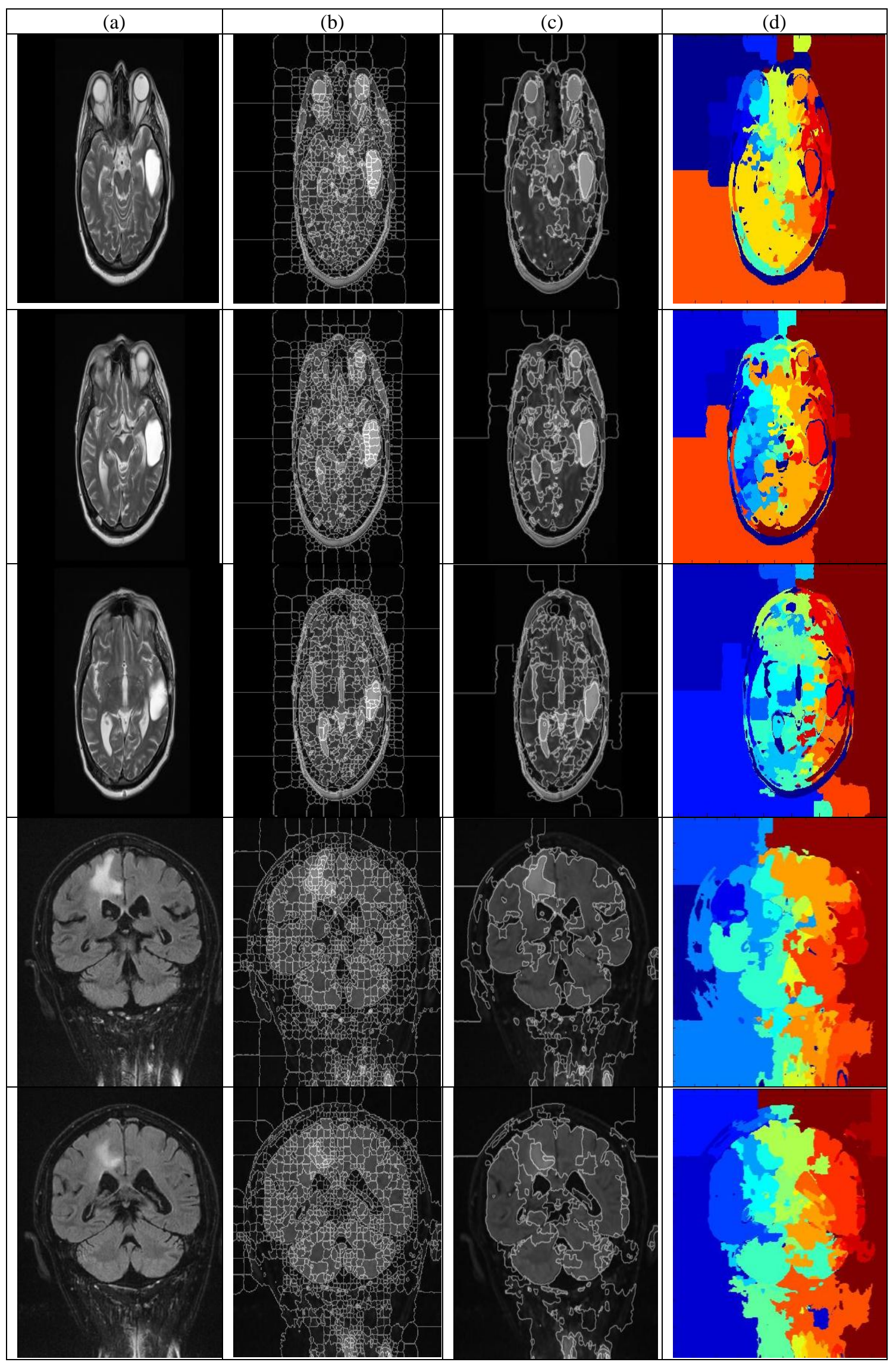

Volume 6 Issue 7, July 2017

www.ijsr.net

Licensed Under Creative Commons Attribution CC BY 
International Journal of Science and Research (IJSR)

ISSN (Online): 2319-7064

Index Copernicus Value (2015): 78.96 | Impact Factor (2015): 6.391

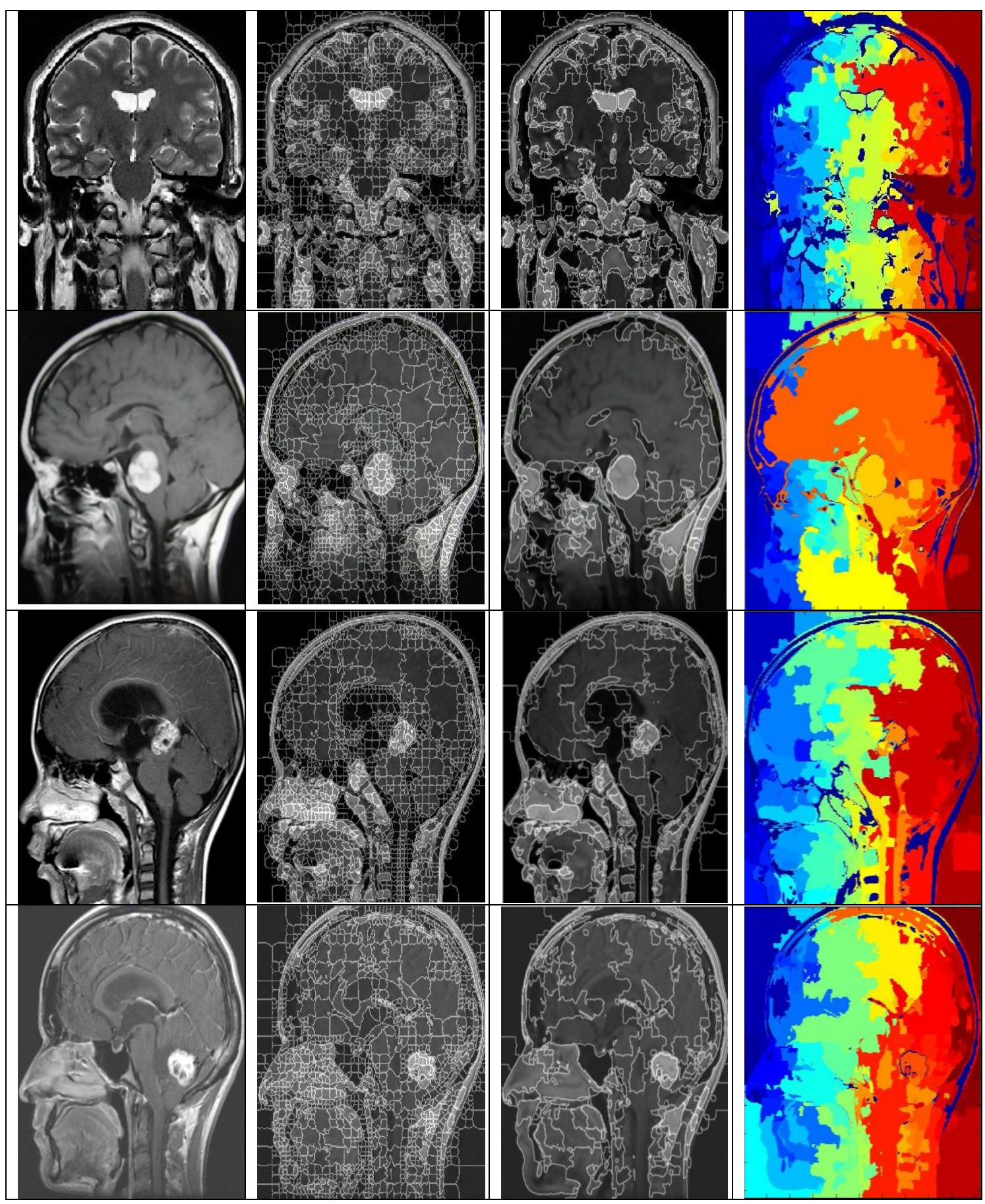

Figure 3: a) Original MRI Images. b) Split resulted regions c) Merge resulted regions d) Segmented image using Split and Merge.

Volume 6 Issue 7, July 2017

www.ijsr.net

Licensed Under Creative Commons Attribution CC BY 